\title{
Improvement of Mechanical Properties of Noil Hemp Fiber Reinforced Polypropylene Composites by Resin Modification and Fiber Treatment
}

\author{
Zili Yan, ${ }^{1,2}$ Jianchun Zhang, ${ }^{2,3}$ Hua Zhang, ${ }^{2,3}$ and Hao Wang ${ }^{1}$ \\ ${ }^{1}$ Centre of Excellence in Engineered Fibre Composites, University of Southern Queensland, Toowoomba, QLD 4350, Australia \\ ${ }^{2}$ Special High Value Biomass Industry and Technology Innovation Strategic Alliance, Beijing 100082, China \\ ${ }^{3}$ The Research Centre of China-Hemp Materials, Beijing 100082, China
}

Correspondence should be addressed to Zili Yan; yan_zili@sina.com and Hao Wang; hao.wang@usq.edu.au

Received 31 May 2013; Revised 15 August 2013; Accepted 23 August 2013

Academic Editor: Hazizan Md Akil

Copyright (C) 2013 Zili Yan et al. This is an open access article distributed under the Creative Commons Attribution License, which permits unrestricted use, distribution, and reproduction in any medium, provided the original work is properly cited.

The present study aims to improve the reinforcement of hemp fibre to polypropylene (PP) by simple resin modification and fibre treatment. Maleic anhydride grafted polypropylene (MAPP) was used as resin modifier by direct mixing with PP, and hydrophobically modified hydroxyethyl cellulose (HMHEC) was used as fibre treatment reagent by immersing fibre into its aqueous solution. The influences of fibre content, resin modification, and fibre treatment on the mechanical properties (tensile, flexural, and impact strengths) of composites were investigated. The change of interfacial bonding between fibre and resin in composites caused by MAPP and HMHEC was studied by scanning electron microscopy and dynamic mechanical analysis. Resin modification and fibre treatment were effective to enhance the mechanical properties of the composites. The improvement in interfacial bonding is quantitatively evaluated with adhesion factor.

\section{Introduction}

Glass fibres are the most widely used reinforcement to plastics due to their low cost and satisfactory effectiveness. However, their drawbacks are also evident, including the health risk when handling and nonbiodegradability when disposed $[1,2]$. Using natural fibres, especially plant fibres, as replacement of glass fibres can overcome the abovementioned problems with additional advantages, including cost-effectiveness, low density, high specific strength, and their availability as renewable resources [3-5]. So far, natural fibres have been used to replace glass fibres in automotive industry but mostly in nonstructural components $[6,7]$. The first and most important reason for the limited application of natural fibre composites is due to the poor interfacial bonding between the hydrophilic natural fibres and the hydrophobic resins, which means the poor shear stress transferring ability at microscale and the unsatisfactory mechanical properties at macroscale in the composites [8,9]. Additional disadvantages arisen from this drawback are apparent fibre breakage and unavoidable thermal degradation when internal mixing or extruding is used to fabricate compound of natural fibre and plastic because strong shearing force and long compounding time are necessary to realize homogeneous fibre distribution [10-12].

Many studies have been focused on resin modification and fibre treatment to improve interfacial bonding. For thermoplastic matrix composites, a lot of resin modifiers have been developed and shown positive effect on interfacial bonding and mechanical properties [13-15]. The most effective modifier is maleic grafted polymer in which the maleic anhydride can react with the hydroxygen group on natural fibre surface, and the main chain of modifier entangles with the matrix [15]. However, the overall mechanical properties of the composites are still lower than corresponding glass fibre reinforced composites [16]. Fibre surface treatment is another approach to achieve improved mechanical performance. Various fibre treatments have been developed using alkali, isocyanate, silane, and acrylic acids, permanganate, and so forth $[17,18]$. Nevertheless, much water is consumed to wash 
TABLE 1: The chemical composition of NHF.

\begin{tabular}{lccccc}
\hline Components & Cellulose, wt $\%$ & Lignin, wt $\%$ & Hemicellulose, wt $\%$ & Pectin, wt $\%$ & Others, wt $\%$ \\
\hline NHF & 91.9 & 1.7 & 3.5 & 1.2 & 1.7 \\
\hline
\end{tabular}

Note: the chemical composition test was based on dry hemp fibre.

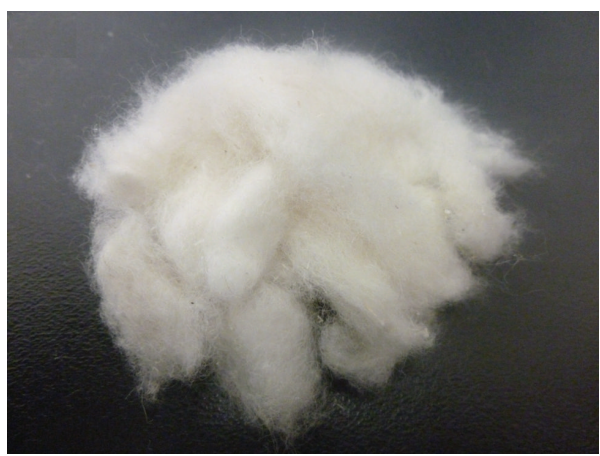

(a)

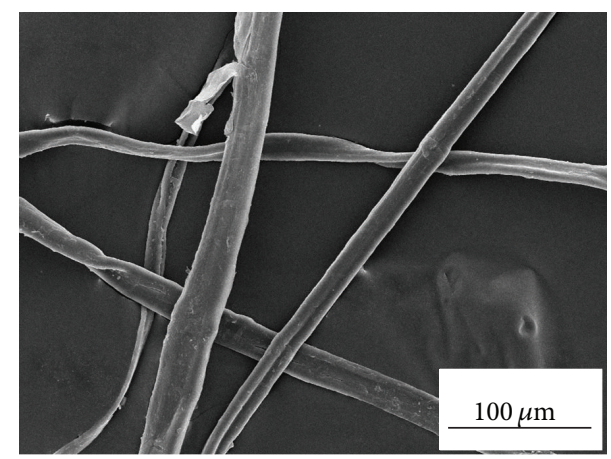

(b)

FIgURE 1: Photo and micrograph of NHF.

the alkali treated fibre to neutral $\mathrm{PH}$, and many of other treatments are undertaken in pure organic solvent or mixed solvent containing organic solvent, which have the problems of disposal of chemicals after treatment [19]. Caulfield et al. [20] used aqueous emulsion of paper sizing agents (alkyl ketene dimer and alkenyl succinic anhydride) to treat cellulose fibre. The treatment improved the hydrophobicity of the fibre and the compatibility between the fibre and the matrix but did not show any improvement in mechanical properties of the composites.

This research is an attempt aiming to develop simple resin modification and fibre treatment route to improve mechanical properties of hemp fibre reinforced polypropylene composites. The fibre treatment was carried out simply by immersing fibre into aqueous solution of hydrophobically modified hydroxyethyl cellulose (HMHEC) followed by oven drying. Resin modification was achieved by applying maleic anhydride grafted polypropylene (MAPP) to Polypropylene (PP).

\section{Experimental Section}

2.1. Raw Materials. Noil hemp fibre (NHF) was obtained from China-Hemp Industrial Investments and Holding Co., Ltd. (Yunnan Province, China). It is an overdegummed hemp fibre by-product in hemp fibre production for textile purpose, with a length shorter than the requirement for textile industry. The chemical composition and microstructure of NHF are shown in Table 1 and Figure 1, respectively. It mainly consists of cellulose (91.9 wt\%) and has low content of pectin, hemicellulose, and lignin. The fibre has smooth surface and an average diameter of $20-30 \mu \mathrm{m}$.

Polypropylene (PP, M800E, Sinopec Shanghai petrochemical Co. Ltd., China) with melt flow index of $8.0 \pm$ $1.5 \mathrm{~g} / 10 \mathrm{~min}$ was chosen as a matrix. MAPP with MA content of 1.0 wt\% (Bondyram 1001, Polyram Ram-On Industries, Israel) was used as a modifier to the resin. HMHEC
(NATROSOL Plus 330, Hercules Chemicals Co. Ltd., China) was used as a fibre treatment reagent. Antioxidant and antiUV agents were applied as additives to the composites.

2.2. Composites Preparation. HMHEC powder was added into stirred water and then kept for $24 \mathrm{~h}$ to get a semitransparent aqueous solution with $0.5 \mathrm{wt} \%$ of HMHEC. Then NHF was immersed in the solution for $0.5 \mathrm{~h}$ with a fibre/solution weight ratio of $1 / 5$. After that, the treated NHF was taken out and heated to $60^{\circ} \mathrm{C}$ for $12 \mathrm{~h}$ and then to $105^{\circ} \mathrm{C}$ for $8 \mathrm{~h}$ to remove water/moisture before it was used for composite fabrication. When no fibre treatment was applied to NHF, it was heated to $105^{\circ} \mathrm{C}$ and kept for $8 \mathrm{~h}$ to remove moisture before mixing with PP.

After the internal mixer was preheated to $170-180^{\circ} \mathrm{C}$, PP, antioxidants $(0.3 \%)$, and an anti-UV agent $(0.3 \%)$ were added into it and mixed for $15 \mathrm{~min}$ to make the resin melt with a rotor speed of $50 \mathrm{rpm}$. Then NHF was added into the mixer to mix with resin melt for $10-15 \mathrm{~min}$ at the same temperature and rotor speed. After that, the composite melt was sheeted using an open mill. The cooled sheets were then crushed into granule of $3-7 \mathrm{~mm}$ in size by a crusher. The granules were finally moulded into specimens at 170$175^{\circ} \mathrm{C}$ for mechanical tests by an injection moulding machine. Composites with 10-40 wt $\%$ NHF were fabricated to evaluate the influence of fibre content on the mechanical properties of composites. Composites with MAPP modification to PP and HMHEC treatment to NHF were also fabricated. For those two composites, MAPP was added into the internal mixer together with PP, and the contents of MAPP and NHF were fixed at $5 \mathrm{wt} \%$ and $30 \mathrm{wt} \%$, respectively.

2.3. Characterizations. In accordance with ISO 527-2-1993, the tensile strength test was performed on dumbbell-shaped specimens of Type 1A using a multifunctional machine (AG-2000A, Shimadzu Corp., Japan) with a loading rate 


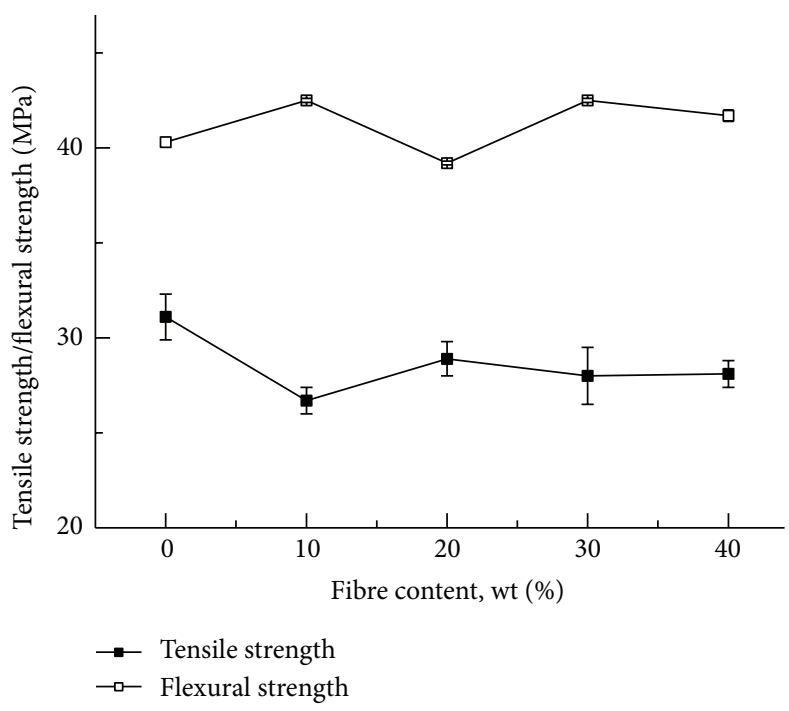

(a)

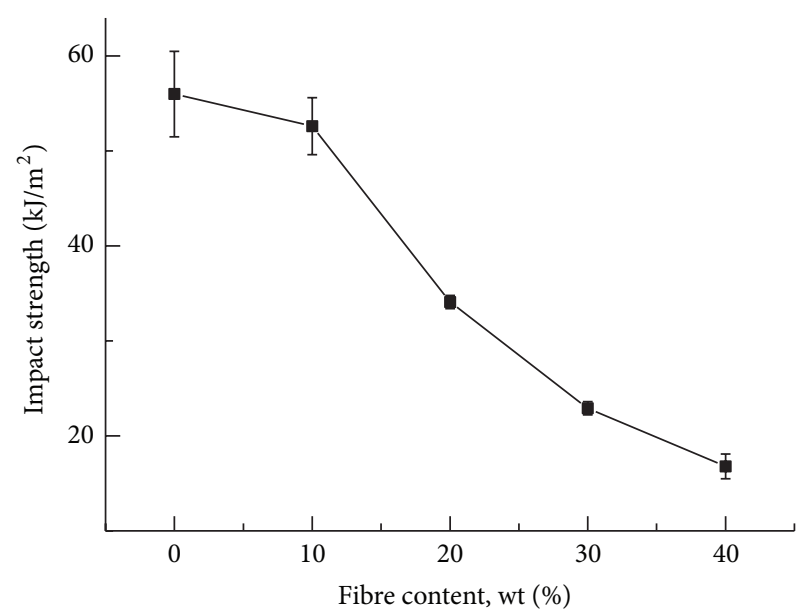

(b)

FIGURE 2: The influence of fibre content on mechanical properties of NHF/PP composites.

of $10 \mathrm{~mm} / \mathrm{min}$ and a $10 \mathrm{kN}$ load cell at room temperature. The standard samples with nominal dimensions of $80 \mathrm{~mm} \times$ $10 \mathrm{~mm} \times 4 \mathrm{~mm}$ were used to measure the flexural strength using the above-mentioned MPa multifunctional machine according to ISO 178-2001 with a loading rate of $3 \mathrm{~mm} / \mathrm{min}$. The unnotched Charpy impact strength test was conducted on standard samples with nominal dimensions of $80 \mathrm{~mm}$ $\times 10 \mathrm{~mm} \times 4 \mathrm{~mm}$ with an impact tester (XCJ24, Chengde Testing Machine Co., Ltd., China) in accordance with ISO 179-2000. All the results were based on average value of at least five specimens.

A scanning electron microscopy (SEM, JSM-646-LV, Shimadzu Corp., Japan) was used to observe the microstructure of the fracture surface of tensile test specimens and fibrematrix interface adhesion. The acceleration voltage is $20 \mathrm{kV}$. The fracture surface of the specimens was lightly sputter coated with carbon before SEM observation. Dynamic mechanical analyzer (DMA, Q-800, TA instrument, USA) was used to evaluate the damping coefficient and the adhesion factor of different composites. Rectangular specimens of $60 \mathrm{~mm} \times 10 \mathrm{~mm} \times 3.5 \mathrm{~mm}$ were used for this test, which were cut and sanded from injection moulded specimens for flexural/impact strength tests. The tests were carried out under a dual cantilever mode at a fixed frequency of $1 \mathrm{~Hz}$ in a temperature range of $30-145^{\circ} \mathrm{C}$. The deflection amplitude was $15 \mu \mathrm{m}$, and the heating ramp rate was $3^{\circ} \mathrm{C} / \mathrm{min}$.

\section{Results and discussion}

3.1. Effect of Fibre Content on Mechanical Properties of the Composites. Fibre content has important influence on the overall performance of composites. Figure 2 shows the effect of NHF content on mechanical properties of the composites without resin modification and fibre treatment. Tensile strength is decreased to $26.7 \mathrm{MPa}$, when fibre content is $10 \mathrm{wt} \%$ compared with neat PP (31.1 MPa). Further increasing fibre content to $40 \mathrm{wt} \%$ has negligible effect on tensile strength of the composites. Flexural strength in NHF/PP almost keeps constant with increasing the NHF from 0 to $40 \mathrm{wt} \%$. Comparing to the tensile strength of composites, the standard deviation of flexural strength is so small that it is invisible. The impact strength of $\mathrm{PP} / \mathrm{NHF}$ decreases with increasing the fibre from $56 \mathrm{KJ} / \mathrm{m}^{2}$ in neat $\mathrm{PP}$ to $16.8 \mathrm{KJ} / \mathrm{m}^{2}$ in $\mathrm{PP} / \mathrm{NHF}$ (40 wt\%). The overall mechanical properties of the composites are lower than neat PP. As has been stated in the pieces of literature $[21,22]$, the low adhesion between the hydrophilic natural fibre and hydrophobic PP is the reason why direct compounding of fibre and PP cannot exhibit fibre reinforcement.

3.2. Effect of Resin Modification and Fibre Treatment. Fibre treatment and resin modification are necessary to achieve the benefit from fibre to reinforce plastics $[15,23]$. Figure 3 shows the influence of resin modification with MAPP and fibre treatment with HMHEC on the mechanical properties of the composites with $30 \mathrm{wt} \%$ fibre. Tensile, flexural, and impact strengths of the composites with MAPP (NHF/PP/MAPP) are $39.3 \mathrm{MPa}, 62.6 \mathrm{MPa}$, and $33 \mathrm{KJ} / \mathrm{m}^{2}$, respectively. Compared to the composites without MAPP (NHF/PP), the improvements by MAPP addition are $40 \%, 47 \%$, and $44 \%$, respectively. The combination of fibre treatment and resin modification (HMHEC/NHF/PP/MAPP) leads to further improvement in tensile strength and impact strength but shows minimal effect on flexural strength. The finial composites show a tensile strength of $44.5 \mathrm{MPa}$ (13\% improvement compared to resin modified composites), a flexural strength of $60.9 \mathrm{MPa}$ (3\% decrease), and an impact strength of $39.3 \mathrm{~kJ} / \mathrm{m}^{2}$ (19\% improvement).

3.3. Fracture Surface of Composites. Figure 4 shows fracture surfaces of the three composites, unmodified, resin modified, 


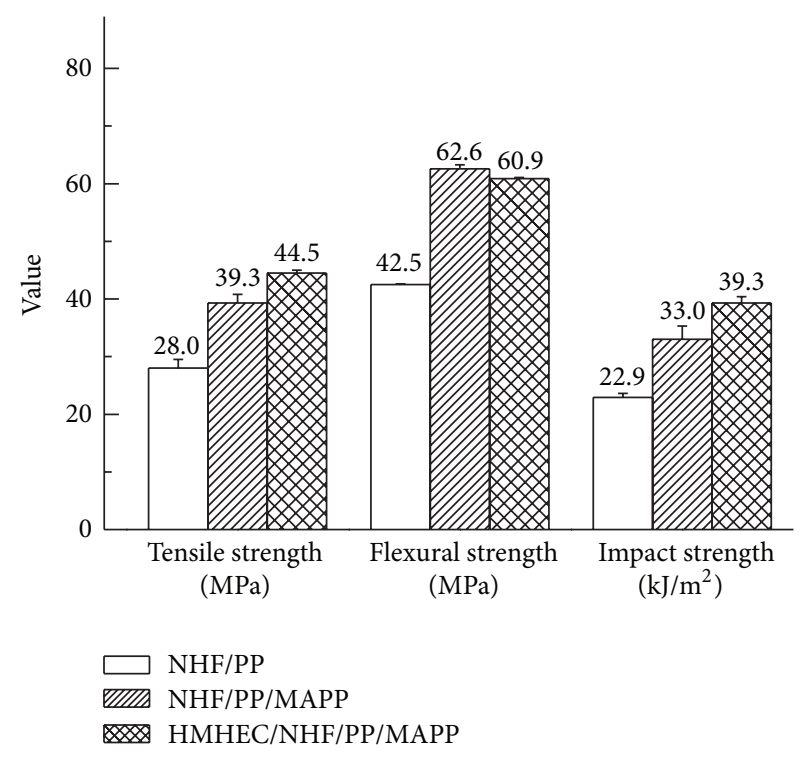

FIGURE 3: Influence of resin modification with MAPP and fibre treatment with HMHEC on the mechanical properties of the composites.

and resin modified plus fibre treated. There is a visible difference in fracture surface between the three composites. When no resin modification and no fibre treatment were conducted to the composites (NHF/PP), fibre has a tendency to be pulled out from the matrix, and the extracted fibre length is long (Figure $4(\mathrm{a})$ ). For the composites with $5 \mathrm{wt} \%$ MAPP resin modification (NHF/PP/MAPP) (Figure 4(b)), fibre breakage is the main fracture mechanism, and the extracted fibre is shorter than that in NHF/PP. The fracture surface of composites with both resin modification and fibre treatment (HMHEC/NHF/PP/MAPP) shows similar appearance with NHF/PP/MAPP but less fibre pullout (Figure 4(c)).

The previous difference demonstrates the enhanced interfacial bonding in composites by resin modification and fibre treatment. MAPP has been used as modifier in wood plastic composites to improve interfacial bonding because of the reaction between maleic anhydride of MAPP and hydrogen on fibre surface, together with the entanglement of the PP chain between MAPP and the matrix [2426]. HMHEC for fibre treatment has hydroxyethylcellulose (HEC) as hydrophilic main chain and long-chain alkyl group as hydrophobic graft chain (Figure 5) [27]. The similar structure between HEC and cellulose in NHF allows Van der Waals forces and hydrogen bond between the modifier and NHF. The olefinic structure of the graft chain is miscible with polypropylene. Both the graft chain and the main chain of HMHEC contributed to the improvement of the interfacial bonding of the composites.

3.4. Fibre Matrix Adhesion Factor. Dynamic mechanical analysis (DMA) is a powerful technique to characterize transition and relaxation process of matrix resin and the effect from fibre [28]. $\tan \delta$, damping coefficient from DMA test, is to characterize the energy dissipation of polymers and their composites. It has been commonly employed to determine the interfacial characteristics of composites because interfacial bonding is one of the most significant sources of damping in composites [29]. In general, a perfect interface will transfer the entire load from matrix to fibre and does not therefore contribute to the damping characteristics. Based on previous research with DMA, an adhesion factor $(A)$ is adopted to evaluate the interfacial bonding of composites [30], which can be expressed as

$$
A=\frac{1}{1-V_{f}} \frac{\tan \delta_{c}}{\tan \delta_{m}}-1,
$$

in which $V_{f}$ is fibre volume fraction and $\tan \delta_{c}$ and $\tan \delta_{m}$ represent the damping coefficients of the composite and the matrix, respectively. A low value of adhesion factor is an indication of good interfacial bonding between the fibre and the matrix.

The damping coefficients of neat PP and three composites are shown in Figure $6 . \tan \delta$ of neat PP and its composites increases with the rising of temperature. However, there is an apparent difference between them. Compared with neat $\mathrm{PP}, \mathrm{NHF} / \mathrm{PP}$ has lower $\tan \delta$ at low temperature but higher $\tan \delta$ at high temperature, whereas the composites with resin modification and fibre treatment, NHF/PP/MAPP and HMHEC/NHF/PP/MAPP, have lower $\tan \delta$ in the whole temperature range of the DMA test, which means that the resin modification and the fibre treatment improve the interfacial bonding in composites. Furthermore, the fibre treatment leads to even lower $\tan \delta$ of the composites (HMHEC/NHF/PP/MAPP) at high temperature. Figure 7shows the calculated adhesion factors of three composites. Unmodified composites (NHF/PP) have higher adhesion factor in the whole temperature range. The MAPP resin modification brings about a significant decrease in adhesion factor. When the fibre treatment is combined with resin modification, the adhesion factor is slightly increased at low temperature but noticeably decreased at high temperature.

The results are consistent with the molecular movement theory and the previous research on the dynamic mechanical analysis of composites [31, 32]. Different from single phase of neat resin, the composite is consists of three phases: matrix, fibre, and interfacial phases. Therefore, besides the matrix, both the fibre and the interface affect the comprehensive properties of the composites, including the damping behavior. The fibre behaves more elastically than the matrix resin [31], which leads to the reduced energy dissipation of composites in the whole temperature range of the DMA test. The interfacial bonding in composites affects $\tan \delta$ by restricting the molecular movement of the resin on the fibre surface. In NHF/PP, the poor interfacial bonding between NHF and PP shows limited restriction to the molecular movement of matrix, especially at high temperature. Due to the combined influence by fibre and the interfacial bonding, NHF/PP has lower $\tan \delta$ at low temperature and higher $\tan \delta$ at low temperature than PP. The effect of MAPP in enhancing interfacial bonding has been repeatedly testified in this study and leads to the lower $\tan \delta$ of NHF/PP/MAPP than that of NHF/PP. 


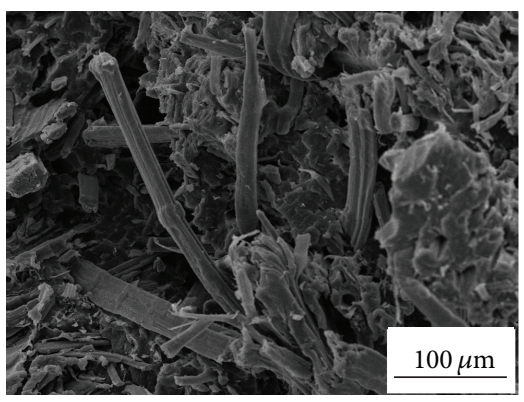

(a)

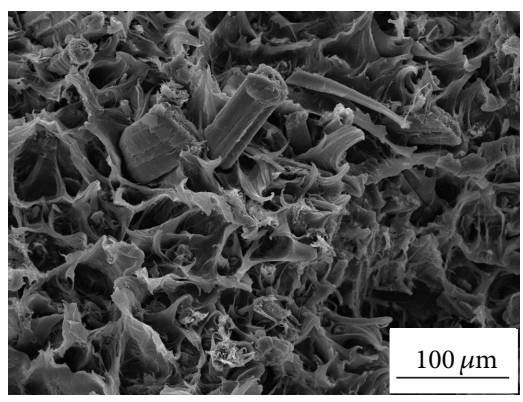

(b)

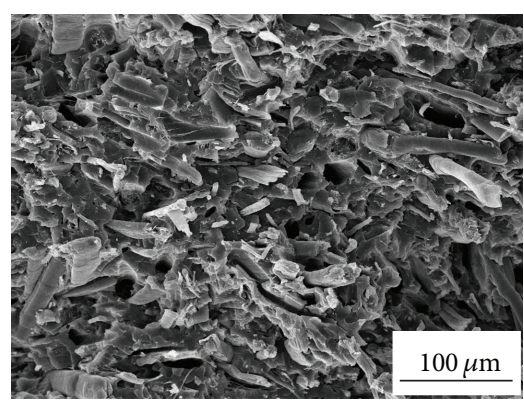

(c)

Figure 4: SEM images of fracture surface of (a) NHF/PP; (b) NHF/PP/MAPP; (c) HMHEC/NHF/PP/MAPP.

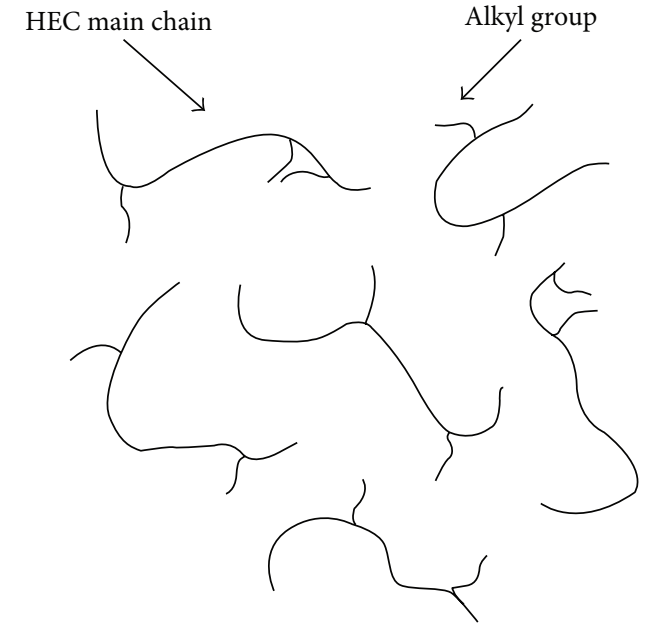

FIGURE 5: Illustration of HMHEC in aqueous solution.
The further decreased $\tan \delta$ in HMHEC/NHF/PP/MAPP at high temperature suggests the effectiveness of fibre treatment in improving the interfacial bonding. Based on the previous discussion, it suggests that $\tan \delta$ of composites at high temperature is more related with interfacial bonding. Figure 7 shows an adhesion factor sequence of NHF/PP > NHF/PP/MAPP > HMHEC/NHF/PP/MAPP at high temperature, which means increased interfacial bonding by resin modification and fibre treatment. Slightly high adhesion factor of HMHEC/NHF/PP/MAPP at low temperature (less than $65^{\circ} \mathrm{C}$ ) compared to NHF/PP/MAPP is also observed Figure 7. Similar result was reported in the research of glass sphere/polyethylene composites [32]. It could be attributed to high molecular mobility of HMHEC due to its small molecular weight compared with PP. It is also noticed that MAPP addition results in more significant reduction of adhesion factor, which indicates that resin modification is more effective in improving the mechanical properties of the hemp/PP composites.

\section{Conclusions}

The incompatibility of hydrophilic hemp fibre and hydrophobic polypropylene leads to poor interfacial bonding between

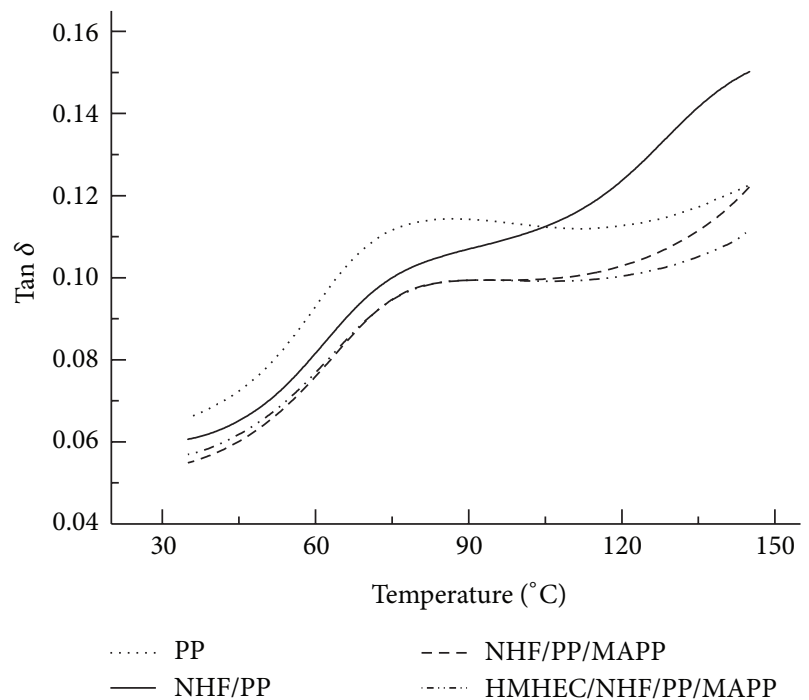

FIgUre 6: Plots of $\tan \delta$ versus temperature of neat PP and its composites.

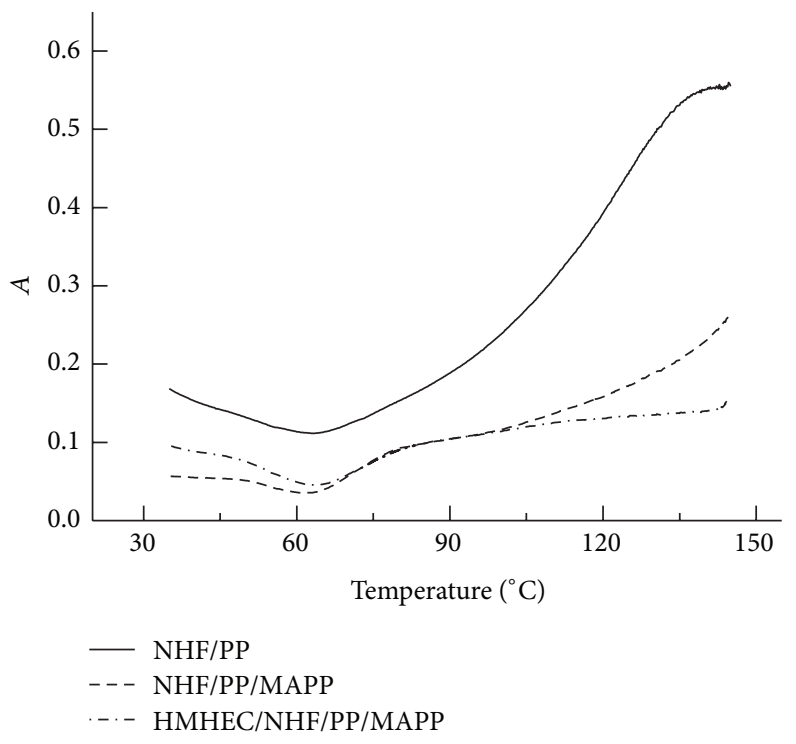

FIGURE 7: Adhesion factor of different composites. 
the fibre and the matrix. Resin modification and fibre treatment are effective in improving the interfacial bonding, which results in improved mechanical properties. MAPP resin modification leads to $40 \%$ improvement in tensile strength, $47 \%$ in flexural strength, and $44 \%$ in impact strength. When combining fibre treatment with resin modification, the mechanical properties are further improved, and the resulted hemp/PP composites achieved a tensile strength of $44.5 \mathrm{MPa}$, a flexural strength of $60.9 \mathrm{MPa}$, and an impact strength of $39.3 \mathrm{KJ} / \mathrm{m}^{2}$. The fracture surface observation indicates that the fracture mechanism is changed from fibre pullout to fibre breakage after the resin modification and fibre treatment. The improvement of interfacial bonding can be quantitatively evaluated by fibre matrix adhesion.

\section{Acknowledgments}

This work has been partially supported by Australia Cooperative Research Centre in Advanced Composite Structures. The authors would like to thank Wenzhou Lisen Enterprises Co., Ltd., for its help in carrying out the experimental work.

\section{References}

[1] P. Asokan, M. Osmani, and A. D. F. Price, "Assessing the recycling potential of glass fibre reinforced plastic waste in concrete and cement composites," Journal of Cleaner Production, vol. 17, no. 9, pp. 821-829, 2009.

[2] M. J. John and S. Thomas, "Biofibres and biocomposites," Carbohydrate Polymers, vol. 71, no. 3, pp. 343-364, 2008.

[3] F. P. La Mantia and M. Morreale, "Green composites: a brief review," Composites Part A, vol. 42, no. 6, pp. 579-588, 2011.

[4] S. N. Monteiro, F. P. D. Lopes, A. S. Ferreira, and D. C. O. Nascimento, "Natural-fiber polymer-matrix composites: cheaper, tougher, and environmentally friendly," JOM, vol. 61, no. 1, pp. 17-22, 2009.

[5] K. G. Satyanarayana, G. G. C. Arizaga, and F. Wypych, "Biodegradable composites based on lignocellulosic fibers: an overview," Progress in Polymer Science, vol. 34, no. 9, pp. 9821021, 2009.

[6] H. Y. Cheung, M. P. Ho, K. T. Lau, F. Cardona, and D. Hui, "Natural fibre-reinforced composites for bioengineering and environmental engineering applications," Composites Part B, vol. 40, no. 7, pp. 655-663, 2009.

[7] M. S. Huda, L. T. Drzal, D. Ray, A. K. Mohanty, and M. Misra, "Natural-fibre composites in the automotive sector," in Properties and Performance of Natural-Fibre Composites, K. L. Pickering, Ed., pp. 256-261, CRC Press, Washington, DC, USA, 2008.

[8] D. Puglia, J. Biagiotti, and J. M. Kenny, "A review on natural fibre-based composites. Part II: application of natural reinforcements in composite materials for automotive industry," Journal of Natural Fibers, vol. 1, no. 3, pp. 23-65, 2004.

[9] M. P. Ho, J. H. WangH Lee, C. K. Ho, K. T. Lau, J. S. Leng, and D. Hui, "Critical factors on manufacturing processes of natural fibre composites," Composites Part B, vol. 43, no. 8, pp. 35493562, 2012.

[10] N. L. Moigne, M. V. D. Oever, and T. Budtova, "A statistical analysis of fibre size and shape distribution after compounding in composites reinforced by natural fibres," Composites Part A, vol. 42, no. 10, pp. 1542-1550, 2011.

[11] A. Etaati, H. Wang, S. Pather, Z. L. Yan, and S. Mehdizadeh A, "3D X-ray microtomography study on fibre breakage in noil hemp fibre reinforced polypropylene composites," Composites Part B, vol. 50, pp. 239-246, 2013.

[12] A. Alemdar, H. Zhang, M. Sain, G. Cescutti, and J. Müssig, "Determination of fiber size distributions of injection moulded polypropylene/natural fibers using X-ray microtomography," Advanced Engineering Materials, vol. 10, no. 1-2, pp. 126-130, 2008.

[13] T. T. Doan, S. L. Gao, and E. Mäder, "Jute/polypropylene composites I. Effect of matrix modification," Composites Science and Technology, vol. 66, no. 7-8, pp. 952-963, 2006.

[14] J. Qian, L. Zhu, J. Zhang, and R. S. Whitehouse, "Comparison of different nucleating agents on crystallization of poly(3hydroxybutyrate-co-3-hydroxyvalerates)," Journal of Polymer Science B, vol. 45, no. 13, pp. 1564-1577, 2007.

[15] T. J. Keener, R. K. Stuart, and T. K. Brown, "Maleated coupling agents for natural fibre composites," Composites Part A, vol. 35, no. 3, pp. 357-362, 2004.

[16] J. L. Thomason, "Why are natural fibres failing to deliver on composite performance?" in Proceedings of the 17th International Conference on Composite Materials (ICCM '17), Edinburgh, UK, July 2009.

[17] X. Li, L. G. Tabil, and S. Panigrahi, "Chemical treatments of natural fiber for use in natural fiber-reinforced composites: a review," Journal of Polymers and the Environment, vol. 15, no. 1, pp. 25-33, 2007.

[18] J. George, M. S. Sreekala, and S. Thomas, "A review on interface modification and characterization of natural fiber reinforced plastic composites," Polymer Engineering and Science, vol. 41, no. 9, pp. 1471-1485, 2001.

[19] X. Yuan, K. Jayaraman, and D. Bhattacharyya, "Effects of plasma treatment in enhancing the performance of woodfibrepolypropylene composites," Composites Part A, vol. 35, no. 12, pp. 1363-1374, 2004.

[20] D. F. Caulfield, J. A. Koutsky, and D. T. Quillen, "Cellulose/polypropylene composites: the use of AKD and ASA sizes as compatibilizers," in Wood-Fiber/Polymer Composites: Fundamental Concepts, Processes, and Material Options, pp. 128-134, 1993.

[21] B. Nyström, R. Joffe, and R. Långström, "Microstructure and strength of injection molded natural fibre composites," Journal of Reinforced Plastics and Composites, vol. 26, pp. 579-599, 2007.

[22] G. W. Beckermann, K. L. Pickering, and N. J. Foreman, "Evaluation of the mechanical properties of injection moulded hemp fibre reinforced polypropylene composites," Advanced Materials Research, vol. 29-30, pp. 303-306, 2007.

[23] J. George, M. S. Sreekala, and S. Thomas, "A review on interface modification and characterization of natural fiber reinforced plastic composites," Polymer Engineering and Science, vol. 41, no. 9, pp. 1471-1485, 2001.

[24] P. Niu, B. Liu, X. Wei, X. Wang, and J. Yang, "Study on mechanical properties and thermal stability of polypropylene/hemp fiber composites," Journal of Reinforced Plastics and Composites, vol. 30, no. 1, pp. 36-44, 2011.

[25] Z. Y. Sun, H. S. Han, and G. C. Dai, "Mechanical properties of injection-molded natural fiber-reinforced polypropylene composites: formulation and compounding processes," Journal of Reinforced Plastics and Composites, vol. 29, no. 5, pp. 637-650, 2010. 
[26] P. Mutjé, M. E. Vallejos, J. Gironès et al., "Effect of maleated polypropylene as coupling agent for polypropylene composites reinforced with hemp strands," Journal of Applied Polymer Science, vol. 102, no. 1, pp. 833-840, 2006.

[27] http://www.in-cosmetics.com/_novadocuments/2234.

[28] P. A. Sreekumar, R. Saiah, J. M. Saiter et al., "Effect of chemical treatment on dynamic mechanical properties of sisal fiberreinforced polyester composites fabricated by resin transfer molding," Composite Interfaces, vol. 15, no. 2-3, pp. 263-279, 2008.

[29] R. Chandra, S. P. Singh, and K. Gupta, "Damping studies in fiber-reinforced composites: a review," Composite Structures, vol. 46, no. 1, pp. 41-51, 1999.

[30] I. Ghasemi and M. Farsi, "Interfacial behaviour of wood plastic composite: effect of chemical treatment on wood fibres," Iranian Polymer Journal, vol. 19, no. 10, pp. 811-818, 2010.

[31] M. Tajvidi, R. H. Falk, and J. C. Hermanson, "Effect of natural fibers on thermal and mechanical properties of natural fiber polypropylene composites studied by dynamic mechanical analysis," Journal of Applied Polymer Science, vol. 101, no. 6, pp. 4341-4349, 2006.

[32] J. Kubat, M. Rigdahl, and M. Welander, "Characterization of interfacial interactions in high density polyethylene filled with glass spheres using dynamic-mechanical analysis," Journal of Applied Polymer Science, vol. 39, no. 7, pp. 1527-1539, 1990. 

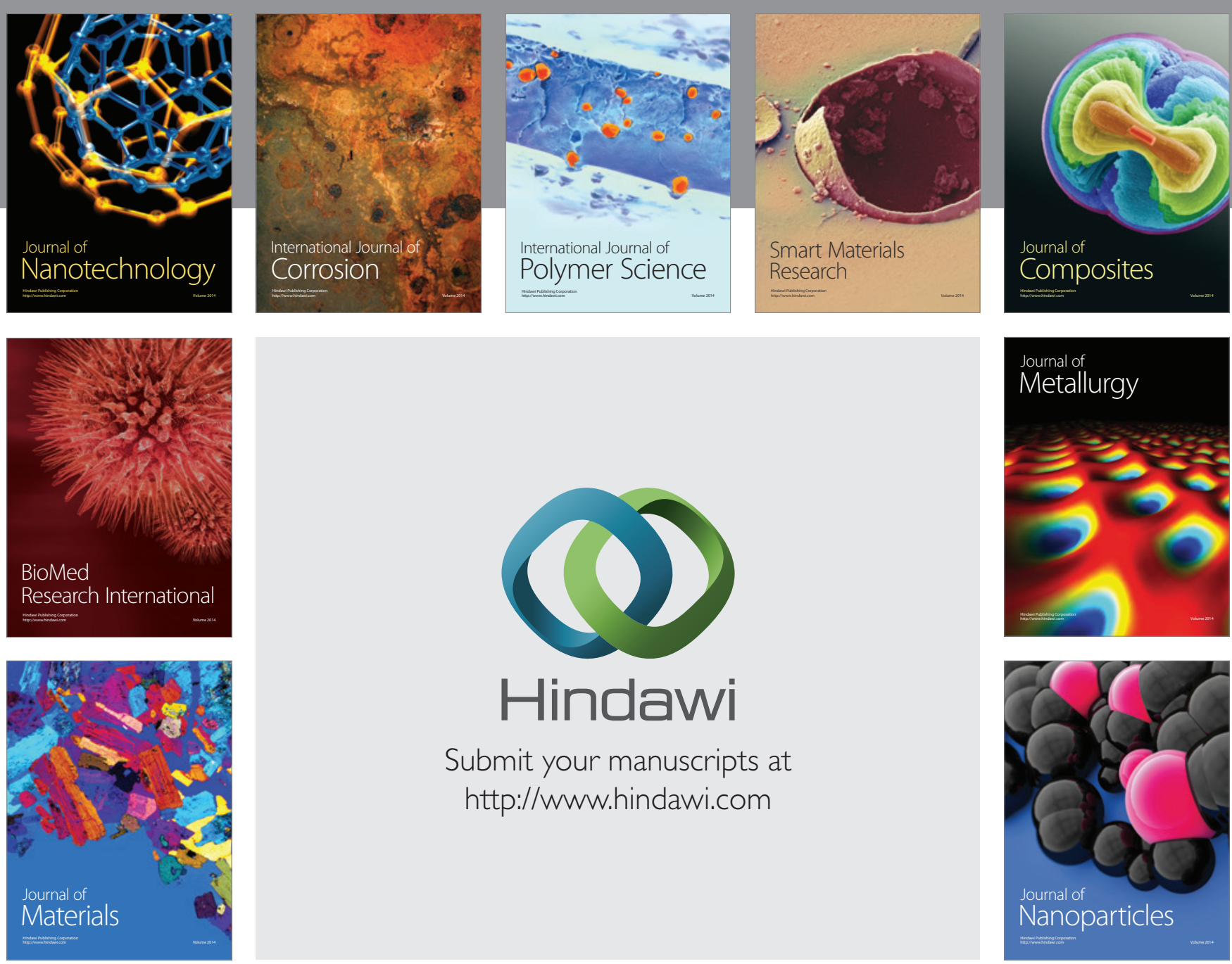

Submit your manuscripts at http://www.hindawi.com
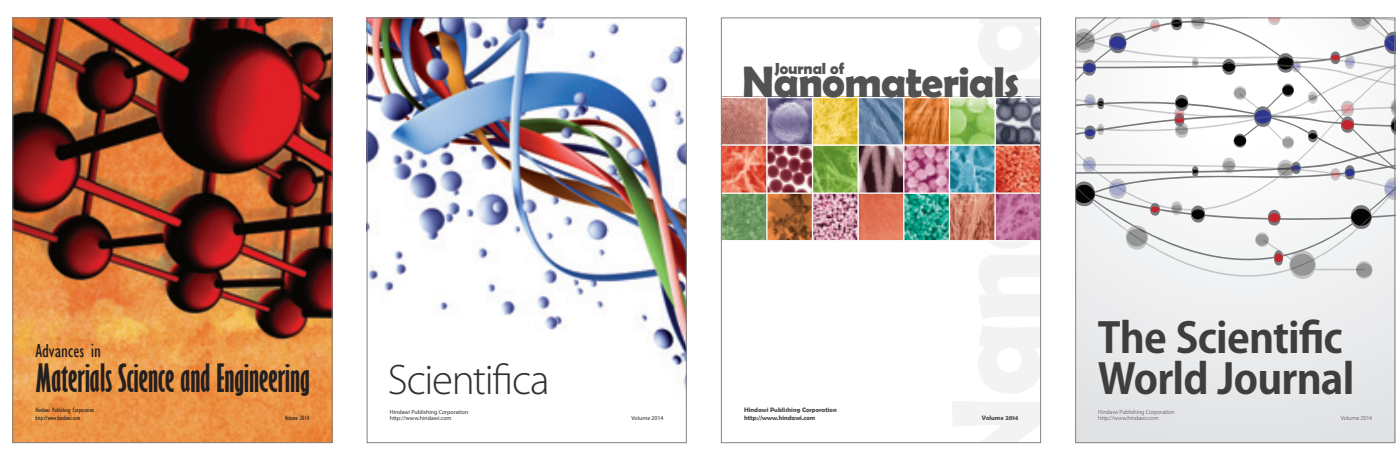

\section{The Scientific World Journal}
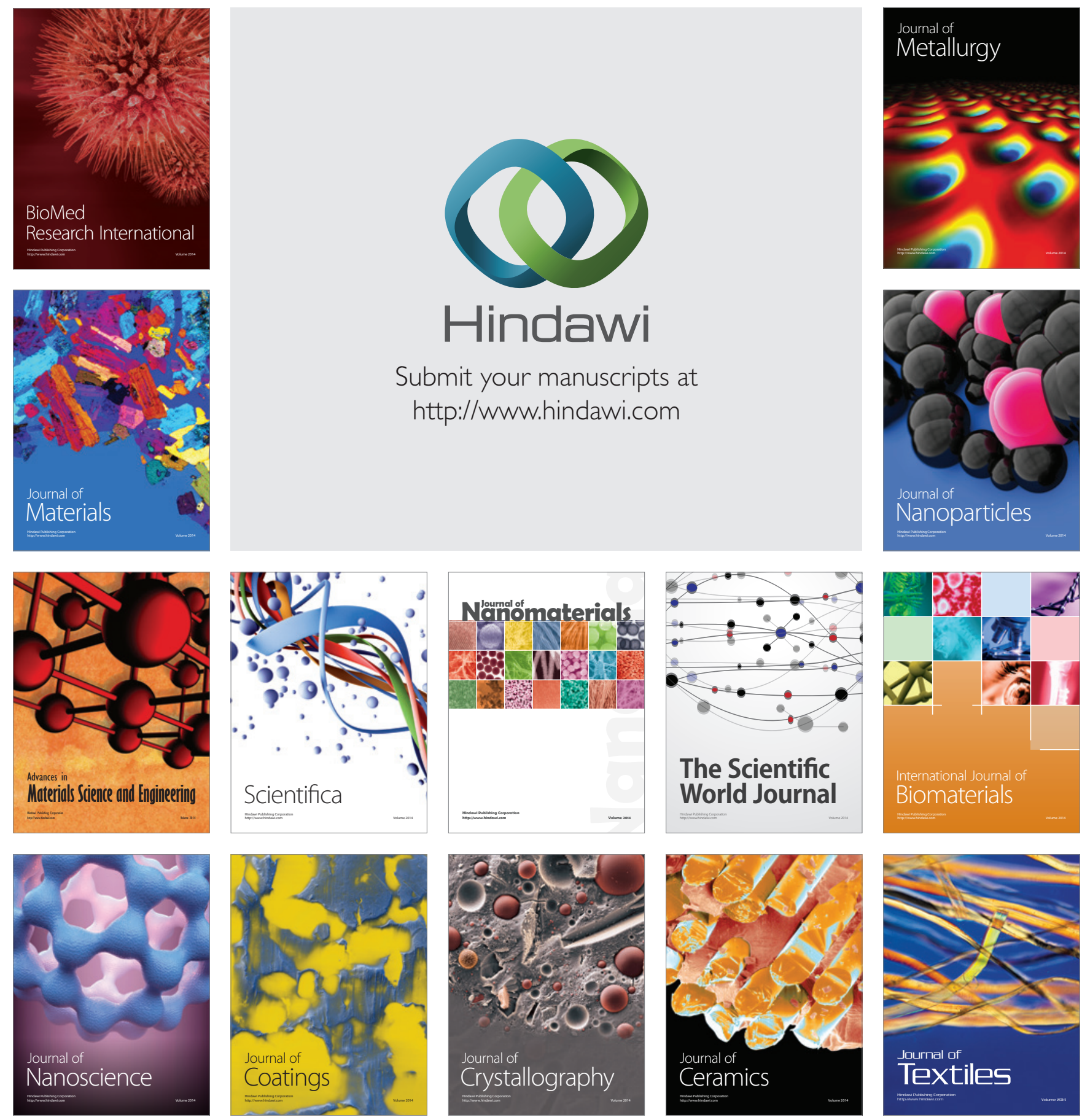